\title{
A randomised controlled trial of topical glycopyrrolate, the first specific treatment for diabetic gustatory sweating
}

\author{
J .E . Shaw ${ }^{1}$, C . A . A bbott ${ }^{1}$, K . Tindle ${ }^{1}$, S. H ollis ${ }^{2}$, A .J . M . B oulton ${ }^{1}$ \\ ${ }^{1}$ Department of Medicine (M7), Manchester Royal Infirmary, Manchester, UK \\ ${ }^{2}$ Medical Statistics Unit, Lancaster University, Lancaster, UK
}

Summary The treatment of gustatory sweating in diabetes mellitus is usually with oral anti-cholinergic drugs, but these frequently lead to unacceptable side effects. Glycopyrrolate is an anti-muscarinic agent that can be applied topically and is efficacious in gustatory sweating occurring in other conditions. In a double-blind placebo-controlled crossover study, we assessed the value of glycopyrrolate in 13 diabetic patients with gustatory sweating. Sweating was measured by a sweat challenge, and diaries recorded by the patients throughout the 2 weeks of each treatment period. Compared to placebo, glycopyrrolate reduced the sweat response to a challenge by $82 \%$ $(p<0.01)$. The frequency of episodes of gustatory sweating during the treatment period was also reduced by $51 \%(p<0.01)$, with a nearly $100 \%$ reduction in the frequency of episodes of severe sweating $(p<0.01)$. In conclusion, topically applied glycopyrrolate is a very effective treatment in reducing both the severity and frequency of diabetic gustatory sweating. [Diabetologia (1997) 40: 299-301]

Keywords Diabetes mellitus, autonomic neuropathy, diabetic neuropathy, diabetic nephropathy, gustatory sweating.
Gustatory sweating was first linked to diabetes mellitus by Watkins in 1971 [1], and is now known to occur quite commonly in patients with either diabetic nephropathy or neuropathy [2]. Although not usually a cause of major morbidity, it can, if severe, disturb eating patterns and occasionally make glycaemic control difficult [3], and is often troublesome and embarrassing. Sweating is controlled by sympathetic cholinergic pathways, and treatment has traditionally involved oral anti-cholinergic drugs, but the acceptability of these to patients is low, because of systemic side effects [1]. Topical anti-muscarinic agents, such as glycopyrrolate, have been demonstrated to be effective in controlling gustatory sweating caused by parotid surgery [4] and a recent case report [3] suggested that glycopyrrolate was equally efficacious in diabetic

Received: 23 July 1996 and in revised form: 24 October 1996

Corresponding author: Dr. J. Shaw, Department of Medicine (M7), Manchester Royal Infirmary, Manchester M13 9WL, UK gustatory sweating. We therefore performed the first double blind crossover placebo controlled study of topical glycopyrrolate in diabetic gustatory sweating.

\section{Patients and methods}

Diabetic patients were invited to take part if they gave a clear history of frequent sweating (of the face, scalp or neck) during or immediately after eating food. At baseline, somatic neuropathy was assessed by the modified neuropathy disability score [5] and the vibration perception threshold at the great toe of the dominant foot using a Neurothesiometer (Arnold Howell, London, UK). Autonomic neuropathy was assessed with a Medilog 2000 (Oxford Medical Ltd, Abingdon, UK) to measure the mean expiratory:inspiratory heart rate ratio ( $\mathrm{R}-\mathrm{R}$ ratio) during deep breathing. Serum creatinine and 24-h urinary protein loss were also measured at baseline. Glycopyrrolate was made up into a cream using a cetamacrogol A formulation (standard available cream base) to make a strength of $0.5 \%$, and a placebo cream of similar appearance and consistency was also formulated. Treatment with each agent was for 2 weeks, separated by a 1 week washout period. Subjects were randomised to start with either $0.5 \%$ glycopyrrolate or 
placebo, and were instructed to apply the cream to affected areas on alternate days, avoiding contact with eyes, nose and mouth, and not to wash the areas for $4 \mathrm{~h}$ after each application.

There are no well-established simple methods to quantify sweat production and so two methods were devised. Firstly, a sweat challenge was performed at baseline and at the end of each treatment period. Each subject was asked to provide a food that they recognised as causing gustatory sweating. Foods were only used if they would allow consistency between the three tests (e.g. a specific brand of cheese). For the sweat challenge, sweat production was measured at four sites - two on the forehead and one each on the forearm and lower leg. Absorbent dressings (Primapore; Smith \& Nephew Medical Limited, Hull, UK) were cut to a standard size, weighed and then applied to each of the four areas. The subject then ate the food and after $20 \mathrm{~min}$, the dressings were removed and reweighed. Sweat production was calculated as the difference between the mean weight change of the two forehead dressings and the mean weight change of the two limb dressings, and expressed as $\mathrm{mg} / \mathrm{cm}^{2}$. The same weight of the same food was used at each of the three tests for each subject. The coefficient of variation for repeated measurements was $17.6 \%$. Secondly, subjects were asked to keep a diary throughout the study, in which they rated their gustatory sweating for each meal and snack on a $0-10$ visual scale. The study was approved by the Central Manchester Research Ethics Committee, and informed consent was obtained from each patient.

Statistical analysis involved non-parametric standard methods for cross-over studies [6]. Tests for period effect and carryover (treatment-period interaction) were performed by a Mann-Whitney test of the two randomisation groups comparing the difference between the active and placebo periods and the average of the two periods, respectively. The significance of the treatment effect was determined using a Mann-Whitney test comparing the difference between the first and second periods in the two randomisation groups. The size of the treatment effect (half of the estimated difference between the two groups) and its confidence interval were calculated using Minitab (Minitab Inc., State College, Pa., USA).

\section{Results}

Fourteen subjects were recruited. One subject withdrew after a few days of using glycopyrrolate, due to an eczematous reaction, which improved on withdrawal. Results are presented for the 13 subjects who completed the trial. The background clinical data are shown in Table 1, and show that the majority of the patients had both neuropathy and nephropathy. There was no evidence of any period or carryover effects of the drug between treatment periods $(p>0.35)$. For the sweat challenge, cheese was used by 11 subjects and fruit by two. The simple weight change results (Table 2) showed no evidence of significant background sweating. The calculated values for sweat production (after correction for surface area of the dressing) showed a clear treatment effect (Fig. 1), with a median (interquartile range) value on glycopyrrolate of $0.74(0.00-2.96) \mathrm{mg} / \mathrm{cm}^{2}$, being significantly lower than both the value at baseline $7.41(1.48-11.1) \mathrm{mg} / \mathrm{cm}^{2}$, and that on placebo
Table 1. Clinical data of the patients

\begin{tabular}{lll}
\hline & & $\begin{array}{l}\text { Normal } \\
\text { range }\end{array}$ \\
\hline Age (years) & $46 \pm 11$ & \\
Male/female & $12 / 1$ & \\
IDDM/NIDDM & $10 / 3$ & \\
Vibration perception threshold (volt) & $28.1 \pm 14.9$ & $<25$ \\
Neuropathy disability score & $5.7 \pm 3.5$ & $<4$ \\
Expiratory: inspiratory R-R ratio & $1.10(1.04-1.14)$ & $>1.20$ \\
Creatinine ( $\mu$ mol/l) & $164.5(130-186)$ & $50-120$ \\
24-h urine protein (gram) & $0.40(0.10-1.05)$ & $<0.15$ \\
\hline
\end{tabular}

Values given as mean \pm SD, median (interquartile range) and numbers

Table 2. Sweat production in response to food, measured as weight changes (mg) of absorbent dressings at each of four sites

\begin{tabular}{lll}
\hline & Glycopyrrolate & Placebo \\
\hline Forehead 1 & $10(0-30)^{\mathrm{a}, \mathrm{b}}$ & $30(16-30)^{\mathrm{b}}$ \\
Forehead 2 & $10(0-40)^{\mathrm{a}, \mathrm{b}}$ & $60(10-60)^{\mathrm{b}}$ \\
Arm & $0(0-0)$ & $0(0-0)$ \\
Leg & $0(0-0)$ & $0(0-0)$ \\
\hline
\end{tabular}

Values given are median (interquartile range). ${ }^{\text {a }} p<0.05$ vs placebo; ${ }^{\mathrm{b}} \mathrm{p}<0.05 \mathrm{vs}$ arm and leg

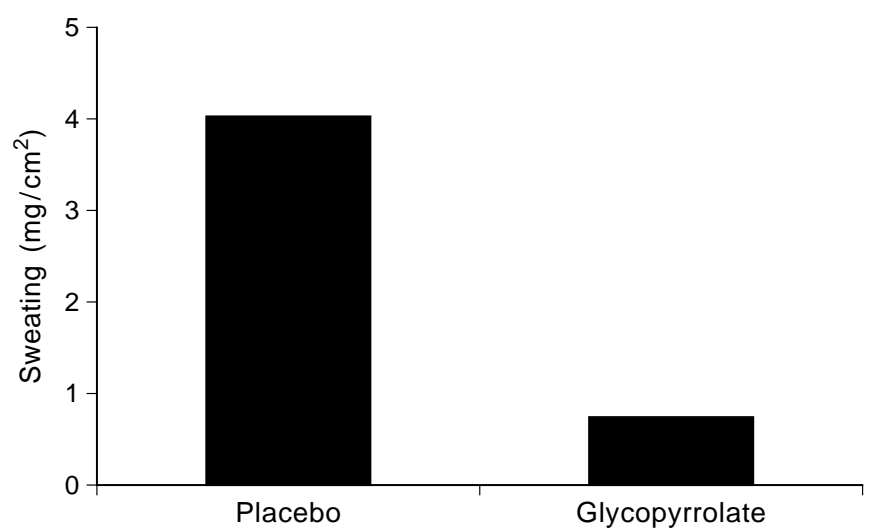

Fig. 1. Results of the sweat test at the end of each treatment period. Median values shown. Placebo vs glycopyrrolate $\mathrm{p}=0.008$

$4.07(1.85-10.37) \mathrm{mg} / \mathrm{cm}^{2}$ ( glycopyrrolate vs placebo $-p=0.008$, placebo vs baseline $-p>0.1$ ). In five of the patients, the sweat response to the challenge was completely abolished by glycopyrrolate, while it was only abolished in one patient by placebo. Mean diary scores were calculated for each subject and as the means were not normally distributed, the median of the means was used for group comparisons. Thus, the median (interquartile range) diary score on glycopyrrolate was $0.84(0.15-1.00)$ and on placebo was $2.00(1.11-2.40), p=0.004$. Glycopyrrolate also reduced the frequency of gustatory sweating (median number of scores $>0)$ by $51 \%(13.5[6.5-27.5]$ vs 27.5[15.0-38.75], $p=0.004)$. It had an even more marked effect on the frequency of more severe 
episodes, with an $84 \%$ reduction in scores over three (1.50 [0.0-3.25] vs 9.5[5.0-11.25], $\mathrm{p}=0.002)$ and almost complete abolition of scores over $5(0.0[0.0$ $1.25]$ vs $1.50[0.0-3.25], p=0.002)$. The drug was well tolerated, and apart from the one patient with a local reaction, no other adverse effects were reported. Ten out of the 13 patients completing the trial wished to continue with the drug. The other three subjects did not feel that the sweating interfered with their lives sufficiently to warrant treatment.

\section{Discussion}

Although gustatory sweating is often embarrassing and troublesome, no simple and acceptable (to patients) treatment has previously been shown to be effective among diabetic patients. Glycopyrrolate is a quaternary amine which acts as an anti-muscarinic agent. It does not cross the blood brain barrier and it penetrates biological membranes slowly, and therefore when given topically leads to very few side effects $[4,7]$. It should, however, not be used in patients with narrow angle glaucoma. The efficacy of topical glycopyrrolate has been demonstrated in Frey's syndrome in controlled studies $[4,7,8]$, and the effect of a single application can last for several days [7]. The results from the current study demonstrate major reductions in both the sweating response to a challenge, and the frequency of gustatory sweating. Evidence from other studies suggests that by increasing the strength of the cream to 1 or $2 \%$, the response could be safely improved in some subjects [7,8]. No simple and reliable methods of quantitative measurement of sweat production exist. The starch-iodine test [1] is the most commonly used in the literature $[4,7,8]$, but the results cannot be quantified and although it may be useful for diagnostic purposes, it is not ideal for a controlled study such as this. We therefore employed our own methods. The sweat challenge uses a straightforward means of measuring the response to a particular food, and by using control areas on the arm and leg negates any possible environmental effects of changes in temperature and humidity. The diary results are less objective than the sweat challenge, but under conditions that require treatment solely for the purpose of symptom control, patient perceptions are particularly important. Patients may have been biased towards filling in only those more serious reactions and might retrospectively have mistakenly scored minor reactions as absent sweating. However, in this group of carefully selected and well-motivated patients, we believe that this would have been only a minor effect. The degree of agreement between the diary results and the sweat challenge supports the reliability of the patient reporting.

A number of oral drugs have been used in gustatory sweating. The oral anti-cholinergics propantheline and oxybutynin, as well as the centrally acting alpha 2 blocker clonidine have all been reported to be effective $[1,9,10]$, but their use may be limited by side effects. Although this may be less of a problem with clonidine and oxybutynin, ours and other data [4] demonstrate an excellent side effect profile and patient acceptability for topical glycopyrrolate. Further studies, however, would be of interest to confirm this in the longer term.

Glycopyrrolate is not manufactured as a cream, but is made as a powder (Robinul, Wyeth, Maidenhead, UK) to be made into a solution for its licensed use in palmar and plantar hyperhidrosis. The cream was therefore made locally from the powder using a cetamacrogol A formulation.

In summary, topical glycopyrrolate is an acceptable, safe and effective treatment for diabetic gustatory sweating. It can be used either on a regular basis or, as some patients expressed a preference, prior to social events. Its main limitation is in patients who have significant sweating on the scalp, as it is not possible to apply it beyond the hairline.

\section{References}

1. Watkins PJ (1973) Facial sweating after food: a new sign of diabetic autonomic neuropathy. BMJ 1: 583-587

2. Shaw JE, Parker R, Hollis S, Gokal R, Boulton AJM (1996) Gustatory sweating in diabetes mellitus. Diabet Med 13: 1033-1037

3. Atkin SL, Brown PM (1996) Treatment of diabetic gustatory sweating with topical glycopyrrolate cream. Diabet Med 13: 493-494

4. Hays LL (1978) The Frey syndrome: a review and double blind evaluation of the topical use of a new anti-cholinergic agent. Laryngoscope 11: 1796-1824

5. Young MJ, Boulton AJM, MacLeod AF, Williams DRR, Sonksen PH (1993) A multicentre study of the prevalence of diabetic peripheral neuropathy in the United Kingdom hospital population. Diabetologia 36: 150-154

6. Everitt BS (1994) Statistical methods for medical investigations. Edward Arnold, London, pp 78-89

7. May JS, McGuirt WF (1989) Frey's syndrome: treatment with topical glycopyrrolate. Head and Neck 11: 85-89

8. Hays LL, Novack AJ, Worsham JC (1982) The Frey syndrome: a simple effective treatment. Otolaryngol Head Neck Surg 90: 419-425

9. Sheehy TW (1991) Diabetic gustatory sweating. Am J Gastroenterol 86: 1514-1517

10. Chiddeckel EW (1990) Oxybutynin for diabetic complications. Am Med Assoc 264: 2994 (Letter) 